https://doi.org/10.1007/s00350-020-5715-8

\section{Anmerkung zu BSG, Urt. v. 11.12.2019 - B 6 KA 12/18 R (Hess. LSG)}

\section{Stefan R. Rohpeter}

Die Entscheidung führt die Rechtsprechung zu Fragen der besonderen Kostenbelastungen in Facharztgruppen fort.

Die spezielle Regelung der Altersversorgung hessischer Vertragsärzte durch eine Umlage mag für Vertragsärzte außerhalb Hessens zunächst keine unmittelbare Relevanz entfalten. Die Rechtsprechung reiht sich aber ein in eine darüber hinausgehende Struktur der Honorarverteilung: Besondere Praxis- und Kostenstrukturen begründen eine besondere Beobachtungs-, Reaktions- und Gestaltungspflicht der KÄVen.

$\mathrm{Ob}$ dies im Rahmen der klassischen Honorarverteilung unter dem Stichwort der ,besonderen Praxisschwerpunkte" maßgeblich ist (vgl. BSG, Urt. v. 26.6.2019 - B 6 KA $1 / 18 \mathrm{R}$ ) oder wie vorliegend bei Heranziehung zu Beiträgen der Altersversorgung: Auch wenn dem untergesetzlichen Normgeber ein erheblicher Gestaltungsspielraum zugestanden wird, findet dieser seine Grenze, wenn die Umsätze zwischen den Fachgruppen nicht mehr vergleichbar sind. Jedenfalls bei einem Anteil von $85 \%$ der Sachkosten am Gesamtumsatz, sah das Gericht eine Notwendigkeit zur Differenzierung.

Fraglich ist, wie bei der Ausgestaltung einer umsatzbezogenen Beitragsfestlegung die besonderen Kosten ermittelt und in welchem Umfang sie berücksichtigt werden müssen.

Die Annahme besonderer Kostenanteile setzt nach der Entscheidung des BSG an der Differenz der Kostensätze zwischen den ärztlichen Gruppen oder abgrenzbaren Untergruppen an und legt eine Schwelle von tendenziell $15 \%$ zugrunde, die bezogen auf den ,,(fiktiven) Durchschnittskostensatz" zu ermitteln sind. Da die Kostensätze sich letztlich stets aus den Besonderheiten der Fachgruppen ergeben, liegt es nahe, dass die Bezugsgröße des fiktiven Durchschnittskostensatzes ohne die jeweilige Fachgruppe oder abgrenzbare Untergruppe ermittelt wird, da ansonsten die Besonderheit einiger Fachgruppen den Durchschnitt aller erhöhen würde, wodurch die Abweichung wiederum geringe ausfiele.

In der Konsequenz müssten die Maßnahmen der KÄV auch sicherstellen, dass eine Mehrbelastung von mehr als $15 \% \mathrm{zu}$ vermeiden ist. Es liegt demzufolge nahe, den Wert der Umsätze unter Berücksichtigung der Kostenanteile differenziert nach Arztgruppen oder abgrenzbaren Untergruppen festzulegen. Daraus ergäbe sich eine arztgruppenspezifische Umlagensatzermittlung.

Es dürfte aufgrund der unterschiedlichen Kostensätze der Fachgruppen mit hohem Kostenanteil auch nicht in Betracht kommen, diese in einer besonderen Gruppe zusammenzufassen: Aufgrund der unterschiedlichen Kostenanteile innerhalb dieser Hochkostengruppe würde es wiederum zu möglicherweise starken Abweichungen kommen, die ihrerseits einer besonderen Beobachtung bedürften.

Eine sich daraus für die KÄV ergebende Schwierigkeit ist indes, dass die Kalkulationsgrundlage des EBM nicht kontinuierlich fortgeschrieben wird und es somit an einer verbindlichen Festlegung der Kostenanteile ebenso mangelt wie an einer nachvollziehbaren Ermittlung derselben.

Es wäre verfehlt, die zum Teil überholte Kalkulationsgrundlage des EBM für eine Beurteilung heranzuziehen. Auch das BSG hat auf die tatsächlichen Kosten abgestellt

Rechtsanwalt Stefan R. Rohpeter,

Fachanwalt für Medizinrecht,

Rohpeter Rechtsanwälte,

Wilhelmshöher Allee 262, 34131 Kassel, Deutschland und festgelegt, dass es bei der Ermittlung der Kostenanteile auch nicht ausreicht, auf gesondert ausgewiesene Sachkosten abzustellen. Vielmehr müssen die tatsächlichen Kosten unabhängig vom Abrechnungsweg zugrunde gelegt werden.

Die beklagte KÄV hat nunmehr die Aufgabe, diese Kostenanteile zu ermitteln und daraus ein belastbares, differenziertes System zu entwickeln.

Die Komplexität hat das BSG selbst erkannt und bewusst auf eine feste Frist für die zügige Neuregelung verzichtet. Jedenfalls wäre ein definierter Zeitraum von sechs Monaten zu kurz, für eine adäquate Umsetzung.

\section{Grundsatz der Honorarverteilungsgerechtigkeit im Rahmen einer Konvergenzregelung}

GG Art. 3, 12; SGB V §87 b

Der generelle Ausschluss von „Wachstumsärzten“ von einer zur Vermeidung überproportionaler Honorarverluste eingeführten Honorarstützungsregelung ist mit dem bundesrechtlichen Grundsatz der Honorarverteilungsgerechtigkeit unvereinbar. (Leitsatz des Bearbeiters)

BSG, Urt. v. 30.10.2019-B 6 KA 21/18 R

(Schleswig-Holsteinisches LSG)

Problemstellung: Ist es gerechtfertigt, Ärzte, die sich in einer Wachstumsphase befinden, von einer Regelung zur Begrenzung von nicht selbst verursachten Honorarverlusten auszuschließen, die bereits etablierten Ärzten zu Teil wird?

Zum wiederholten Male musste sich das BSG mit den Regelungen zur Umsetzung der Einführung der Euro-Gebührenordnung und des RLV zum 1.1.2009 befassen. Wie schon in den Entscheidungen v. 2.8.2017 (B 6 KA 7/17 R) und 24.1.2018 (B 6 KA 2/17 R) lag auch diesem Urt. die 1. Ergänzungsvereinbarung zu der vom Landesschiedsamt festgesetzten „Vereinbarung zur Honorierung vertragsärztlicher Leistungen im Jahre 2009" (HVV) der Gesamtvertragspartner in Schleswig-Holstein zugrunde. Diesmal ging es um die Konvergenzregelung zum Ausgleich überproportionaler Honorarverluste und den ausdrücklichen Ausschluss von Ärzten in einer Wachstumsphase hiervon.

Der erweiterte Bewertungsausschuss (EBewA) eröffnete den Partnern der Gesamtverträge über die Regelungen in Teil F, Ziff. 3.7 des Beschl. der 7. Sitzung v. 27./28.8.2008 i.d.F. des Beschl. der 10. Sitzung v 27.2.2009 (hier Teil A Ziff. 1) die Möglichkeit, ein Konvergenzverfahren zum Ausgleich überproportionaler Honorarverluste zu etablieren.

Von dieser Möglichkeit machten die Partner der Gesamtverträge in Schleswig-Holstein in einer 2. Ergänzungsvereinbarung zum HVV v. 3.4.2009 Gebrauch. Hierin war vorgesehen, dass Honorarverluste, welche auf der Umstellung der Honorarsystematik beruhten, im ersten Halbjahr 2009 durch Ausgleichzahlungen auf maximal 7,5\% begrenzt werden. Von der Anwendung dieser Regelung ausdrücklich ausgenommen wurden jedoch Praxen, die sich in der Wachstumsphase befanden.

Eingesandt von Prof. Dr. iur. Thomas Clemens, Kassel; bearbeitet von Rechtsanwalt Dr. iur. Hendrik Zeiß,

Fachanwalt für Medizinrecht und Fachanwalt für Versicherungsrecht, Ehlers \& Feldmeier Rechtsanwälte Partnerschaftsgesellschaft mbB, Elisabethstr. 6, 44139 Dortmund, Deutschland 